\title{
(2) OPEN ACCESS \\ Randomised trial of cord clamping at very preterm birth: outcomes at 2 years
}

\author{
Lindsay Armstrong-Buisseret (1) , ${ }^{1}$ Katie Powers, ${ }^{1}$ Jon Dorling, ${ }^{2}$ Lucy Bradshaw, \\ Samantha Johnson, ${ }^{3}$ Eleanor Mitchell, ${ }^{1}$ Lelia Duley (i) ${ }^{1}$
}

\begin{abstract}
- Additional material is published online only. To view please visit the journal online (http://dx.doi.org/10.1136/ archdischild-2019-316912)

${ }^{1}$ Nottingham Clinical Trials Unit, University of Nottingham, Nottingham, UK

2Division of Neonatal-Perinatal Medicine, Dalhousie University, Halifax, Canada

${ }^{3}$ Department of Health Sciences, University of Leicester, Leicester, UK
\end{abstract}

\section{Correspondence to}

Professor Lelia Duley,

Nottingham Clinical Trials Unit, University of Nottingham, Nottingham NG7 2RD, UK Lelia.Duley@nottingham.ac.uk

Received 24 January 2019

Revised 15 July 2019

Accepted 17 July 2019

Published Online First

1 August 2019

\section{ABSTRACT}

Objective To report outcomes at 2 years corrected age for children of women recruited to a trial comparing alternative policies for timing of cord clamping and immediate neonatal care at very preterm birth.

Design Parallel group randomised (1:1) trial.

Setting Eight UK tertiary maternity units.

Participants Two hundred and seventy-six babies born to 261 women expected to have a live birth before $32^{+0}$ weeks' gestation.

Interventions Deferred cord clamping ( $\geq 2 \mathrm{~min}$ ) and immediate neonatal care with cord intact or immediate ( $\leq 20$ s) clamping and immediate neonatal care after clamping.

Main outcome measure Composite of death or adverse neurodevelopmental outcome at 2 years corrected age.

Results Six babies born after $35^{+6}$ weeks were excluded. At 2 years corrected age, outcome data were not available for a further 52 children, leaving 218 for analysis (115 deferred clamping, 103 immediate clamping). Overall, 24/115 (21\%) children allocated deferred clamping died or had an adverse neurodevelopmental outcome compared with 35/103 (34\%) allocated immediate clamping; risk ratio (RR) 0.61 (95\% Cl 0.39 to 0.96); risk difference (RD) $-13 \%$ (95\% Cl $-25 \%$ to $-1 \%)$. Multiple imputation for missing data gave an RR 0.69 (95\% Cl 0.44 to 1.09) and RD $-9 \%(95 \% \mathrm{Cl}-21 \%$ to $2 \%)$.

Conclusions Deferred clamping and immediate neonatal care with cord intact may reduce the risk of death or adverse neurodevelopmental outcome at 2 years of age for children born very premature. Confirmation in larger studies is needed to determine the real benefits and harms.

Trial registration number ISRCTN21456601.

\section{INTRODUCTION}

Although just $1.1 \%$ of live births in the UK are very preterm (before 32 weeks' gestation), these infants account for $43 \%$ of neonatal deaths. ${ }^{1}$ Those who survive are at increased risk for a range of neurodevelopmental sequelae including cerebral palsy, neurosensory and cognitive impairment, and attention, social and emotional problems. ${ }^{23}$ Such difficulties can have a major impact on a child's health, well-being and academic attainment and may persist into adulthood. ${ }^{4}$ Interventions that could provide even a modest improvement in long-term outcomes would be of substantial benefit to these children and their families.

\section{What is already known on this topic?}

- A short delay in umbilical code clamping may reduce the risk of death before hospital discharge at very preterm birth.

- Immediate neonatal care can be provided with the cord intact, allowing cord clamping to be deferred for longer in babies requiring resuscitation at birth.

- Previous trials have been small, and few have reported outcomes beyond discharge from hospital.

\section{What this study adds?}

- Neurodevelopmental assessment at 2 years corrected age is feasible using a range of strategies, including routine clinical data.

- Deferred clamping with immediate neonatal care, if needed, may reduce the risk of death or adverse neurodevelopmental outcome at 2 years of age.

- Large high-quality trials and meta-analysis of individual participant data from these trials are needed to confirm the true benefits and harms.

Systematic reviews have suggested that deferring clamping of the umbilical cord at preterm birth may reduce the risk of intraventricular haemorrhage (IVH) ${ }^{6}$ and death before hospital discharge. ${ }^{67}$ However, the trials largely excluded infants requiring immediate resuscitation at birth, and for very preterm births, most trials deferred clamping for $60 \mathrm{~s}$ or less. Also, data on long-term safety are sparse. The Cord Pilot Trial compared alternative policies for cord clamping and immediate neonatal care for very preterm births and results to discharge have been reported. ${ }^{8}$ This paper presents follow-up of the children after discharge and the results of neurodevelopmental assessments at 2 years corrected age.

\section{METHODS}

The Cord Pilot Trial was conducted at eight UK hospitals, and the protocol is published. ${ }^{9} 10$ Women expected to have a live birth before $32^{+0}$ weeks' gestation (very preterm) were randomised 1:1 either to deferred cord clamping after at least $2 \mathrm{~min}$ and, if needed, immediate neonatal care with cord intact or to immediate clamping within 


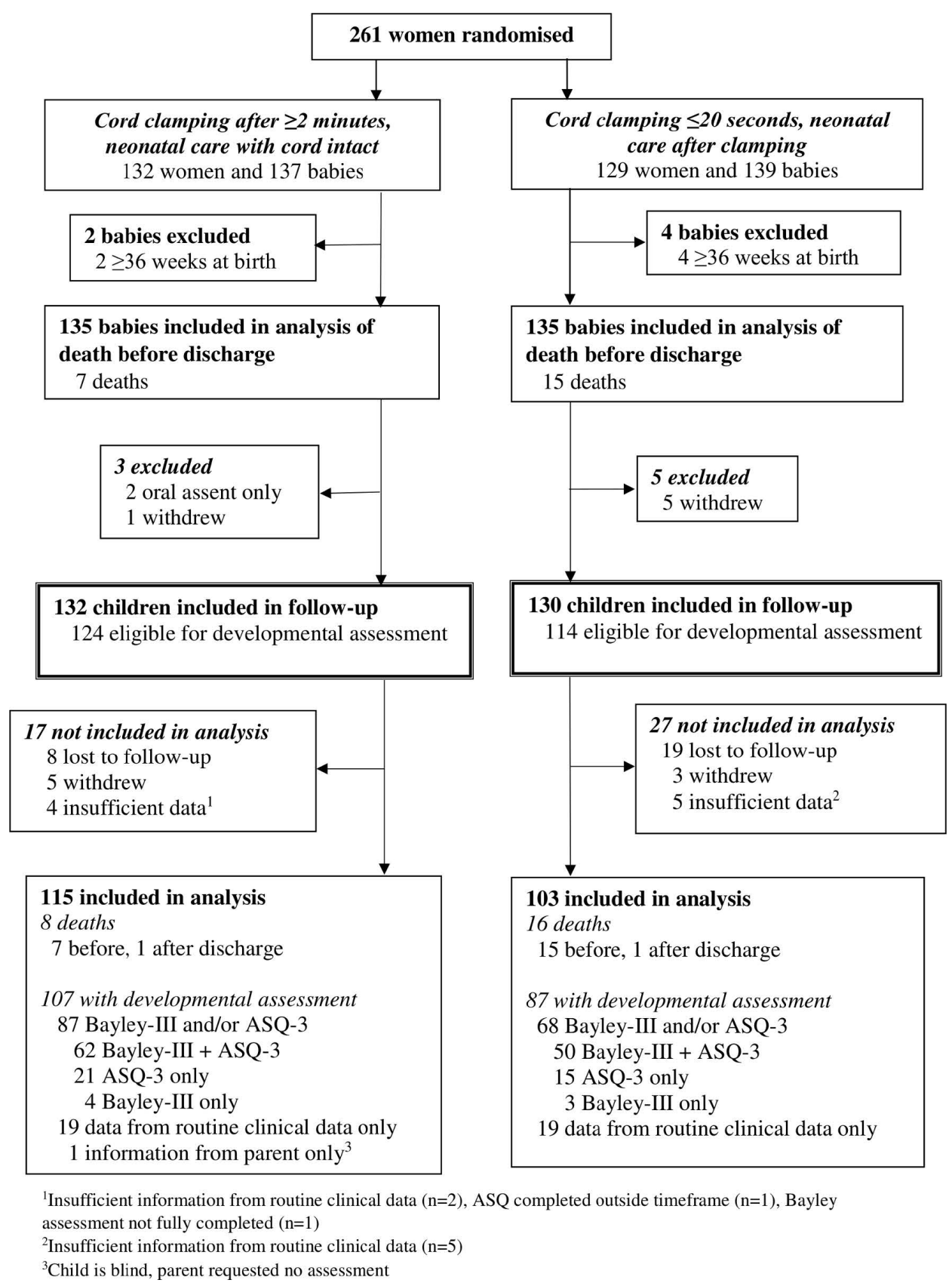

Figure 1 Flow for follow-up of children at 2 years (corrected age). ASQ-3, Ages and Stages Questionnaire-3.

$20 \mathrm{~s}$ and neonatal care after clamping. To ensure women could be recruited when birth was imminent, we included a two-stage consent pathway, with oral assent for recruitment followed by written consent after the birth, alongside the usual one stage pathway (described in detail elsewhere ${ }^{89}$ ). Between March 2013 and February 2015, 261 women were randomised with six excluded as birth was after $35^{+6}$ weeks. ${ }^{8}$ Of the remaining 255 women, 17 had a twin pregnancy, for two of which one fetus died in utero before randomisation, leaving 270 children for the analysis of outcomes at hospital discharge. ${ }^{8}$ Of these, 22 babies died before discharge (including three stillbirths), and a further two died after discharge, giving a total of 24 deaths. Eight children were excluded from follow-up either because the mother had already withdrawn consent $(n=6)$ or she gave oral assent for recruitment, ${ }^{8}$ and subsequent consent for follow-up was not available $(n=2)$. Therefore, 238 children were eligible for assessment (figure 1).

\section{Parent and parent representative involvement}

Parent and parent representative involvement has been reported. $^{8}$ For the follow-up, parent representatives contributed to study design and materials, including the process for contacting families.

\section{Neurodevelopmental assessment of children}

Children were assessed using the parent-completed Ages and Stages Questionnaire-3 (ASQ-3) ${ }^{11}$ and the Bayley Scales of Infant and Toddler Development, Third Edition (Bayley-III). ${ }^{12}$ If neither assessment was conducted, routine clinical data for 2-year outcomes were obtained if available. 
Table 1 For children included in analysis of outcomes to discharge and outcome at 2 years, characteristics at entry and outcomes to discharge

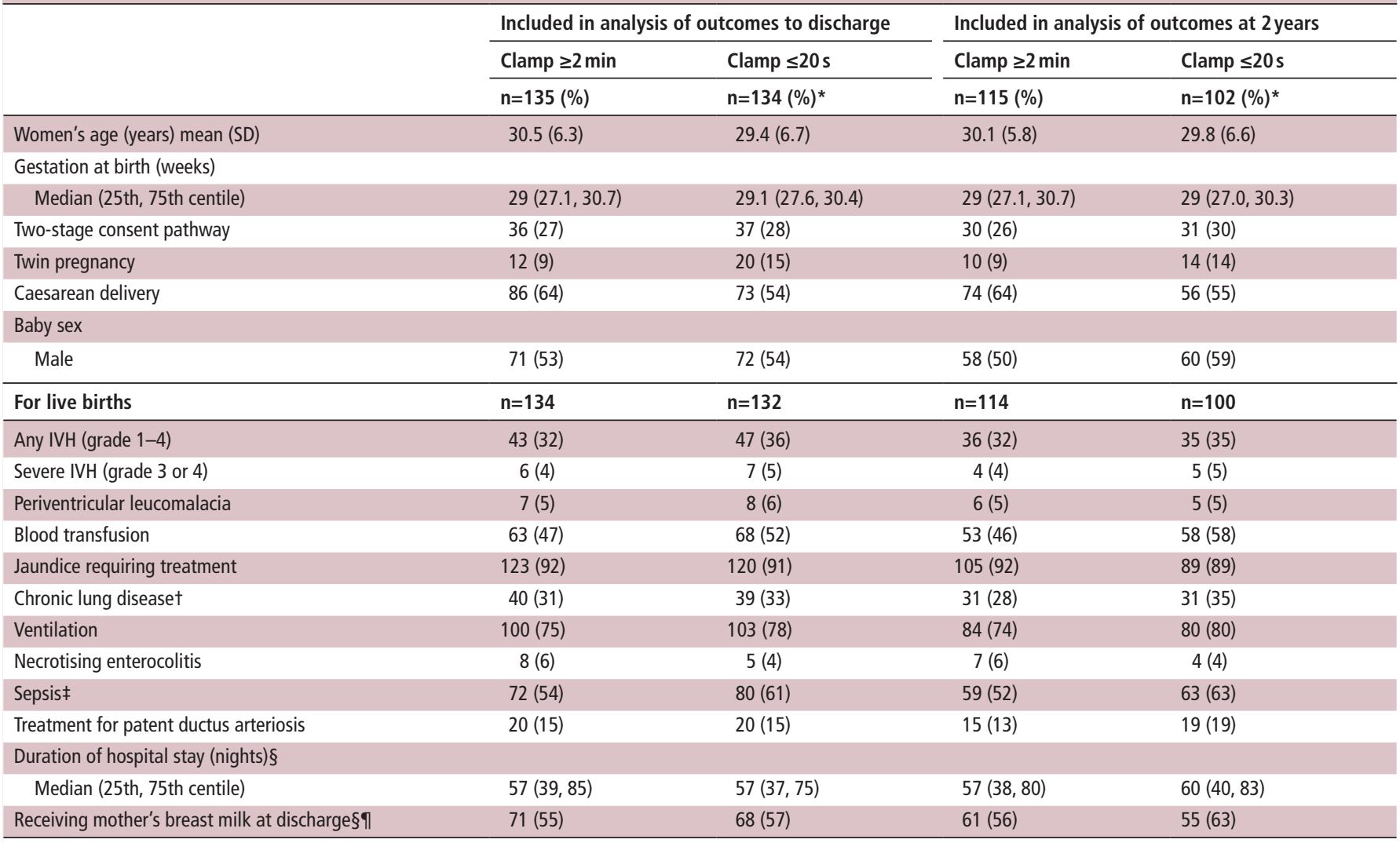

*One woman withdrew (outcome data reported only for death before discharge).

tFor babies surviving to 36 weeks postmenstrual age. $n=129$ clamping $\geq 2 \min , n=120$ clamping $\leq 20$ s for all children and $n=109, n=88$ respectively for children included in analysis at 2 years.

‡Clinical sepsis defined as positive culture and $\geq 5$ days antibiotics or negative culture and $\geq 5$ days antibiotics.

§For babies alive at discharge $n=128$ clamping $\geq 2 \mathrm{~min}, n=120$ clamping $\leq 20$ s for all children and $n=108, n=88$ respectively for children included in analysis at 2 years.

१Receiving breast milk at discharge not known for three babies in the clamp cord $\geq 2$ min group (two included in analysis at 2 years) and one baby in clamp cord $\leq 20 \mathrm{~s}$ group (not included in analysis at 2 years).

$\mathrm{IVH}$, intraventricular haemorrhage.

Shortly before children reached 2 years corrected age, the ASQ-3 was posted to the mother along with an invitation for a Bayley-III assessment. If a completed ASQ-3 was not returned, the family was contacted with up to two reminder letters and a phone call. If no ASQ-3 was returned, parents were asked to complete the appropriate version (24, 27 or 30 months) during the home visit prior to the Bayley-III assessment or during a hospital visit. If $>1$ ASQ-3 was returned, the one completed closest to the date the child was 2 years corrected age was used for analysis.

The ASQ-3 includes 30 questions covering five domains: communication, fine motor skills, gross motor skills, problem solving and personal-social skills. The response to each question is 'not yet', 'sometimes' or 'yes' (scored 0, 5 and 10, respectively). For each domain, scores are summed to produce a total. The domain score is not calculated if $\geq 3$ responses are missing. If one or two responses are missing, the domain score is calculated using the mean of the completed items to impute the missing items. Four further questions to assess hearing, vision and gross motor function were added to the ASQ-3 questions. ${ }^{13}$

The Bayley-III comprises three scales to assess cognitive, language and motor development. ${ }^{12}$ Children were assessed during a home visit by a trained researcher (KP) blind to intervention allocation. Three assessments were video recorded and scored by an independent observer (SJ) to assess inter-rater reliability.

\section{Outcome measures}

The main outcome was a composite of death or adverse neurodevelopmental outcome at 2 years corrected age. Secondary outcomes included the individual components of the composite.

\section{Classification of adverse neurodevelopmental outcome}

Neurodevelopmental outcomes were classified using the Bayley-III scores if available. ${ }^{14} 15$ If the child did not have a Bayley-III assessment, or individual scales could not be completed, ASQ-3 data plus our additional questions on hearing, vision and gross motor function were used. To account for underestimation of developmental delay using the Bayley-III, moderate or severe impairment was based on scores $>1$ SD below the normative mean (scores <85). ${ }^{14} 15$ For each ASQ-3 domain, developmental delay was based on a total score $>2$ SDs below the mean using published norms. ${ }^{11}$

Children were classified as having an adverse neurodevelopmental outcome if they met the criteria for a moderate/severe impairment in any one of five functions: motor, cognitive, speech/ language, hearing or vision (see online supplementary appendix 1). ${ }^{16}$ For motor, this was defined as a Bayley-III gross motor scale score $<7$. If this scale had not been completed, assessment was based on our additional gross motor function questions from the ASQ-3, that is, if the child was unable to walk without assistance or sit without support. For cognitive and speech/language functions, this was defined as having a composite score $<85$ 
Table 2 For children included in follow-up and eligible for neurodevelopmental assessment (at 2 years corrected age), information about the assessment

\begin{tabular}{|c|c|c|}
\hline & $\begin{array}{l}\text { Clamp } \\
\geq 2 \text { min+neonatal care } \\
\text { with cord intact }\end{array}$ & $\begin{array}{l}\text { Clamp } \leq 20 \mathrm{~s} \\
\text { + neonatal care after } \\
\text { clamping }\end{array}$ \\
\hline & $n=124(\%)$ & $\mathrm{n}=114(\%)$ \\
\hline Any neurodevelopment data & $107(86)$ & 87 (76) \\
\hline Bayley-III and/or ASQ-3 & $87(70)$ & $68(60)$ \\
\hline Bayley-III+ASQ-3 & 62 & 50 \\
\hline Bayley-III only & 4 & 3 \\
\hline ASQ-3 only & 21 & 15 \\
\hline Clinical data only & $20(16)^{*}$ & $19(17)$ \\
\hline \multicolumn{3}{|l|}{$\begin{array}{l}\text { Age (corrected) at Bayley-III } \\
\text { (months) }\end{array}$} \\
\hline$<24$ & 2 & 7 \\
\hline$\geq 24$ to $<27$ & 53 & 37 \\
\hline$\geq 27$ to $<30$ & 9 & 9 \\
\hline$\geq 30$ & 2 & - \\
\hline \multicolumn{3}{|l|}{ ASQ-3 version (months) } \\
\hline 24 & 78 & 60 \\
\hline 27 & 3 & 3 \\
\hline 30 & 2 & 2 \\
\hline No neurodevelopmental data & $17(14)$ & $27(24)$ \\
\hline
\end{tabular}

*Information from parent only for one child.

ASQ-3, Ages and Stages Questionnaire-3.

on the relevant Bayley-III scale. If these scales had not been completed, assessment was based on a score below the relevant domain cut-off on the ASQ-3 (problem solving for cognition and communication for language). Hearing and vision were assessed, respectively, as moderate/severely impaired if the child required hearing aids or was deaf, or had difficulty seeing with glasses, could only see light or was blind.

\section{Blinded review of neurodevelopmental outcome}

A blinded review of outcome data was undertaken if: clinical follow-up data only were available, the Bayley-III was not fully completed, the ASQ-3 was not completed within the correct timeframe or the additional questions included on the ASQ-3 about hearing and vision were not completed. For each child, data were assessed independently by two reviewers blind to the allocated group. Discrepancies were resolved by discussion or if needed by a third independent reviewer.

\section{Statistical analyses}

As this was planned as a pilot trial, there was no formal power calculation. ${ }^{910}$ All analyses are based on the groups as randomly allocated (intention to treat) and were carried out using Stata SE 15.1. The main outcome (death or adverse neurodevelopmental outcome) is summarised by allocated group and presented with a risk ratio (RR), risk difference (RD) and 95\% CIs. Each component of the main outcome is also summarised by group.

Multiple imputation using chained equations ${ }^{17}$ was used as a sensitivity analysis to include children with missing outcome data in order to explore their potential impact on the estimate of RR and RD. Variables included in the imputation model were maternal age, gestation at birth, mode of delivery, sex, receiving breast milk at discharge, necrotising enterocolitis, grade of IVH, bronchopulmonary dysplasia, treatment for retinopathy of prematurity and country specific decile of Index Multiple Deprivation. ${ }^{18}$ Thirty datasets were imputed, and estimates were combined using Rubin's rules. ${ }^{17}$ This sensitivity analysis assumed that missing outcomes were missing at random, ${ }^{19}$ that is, conditional on the observed variables the distribution of the observed and missing outcomes are assumed to be the same.

\section{RESULTS}

Of the 238 children eligible for assessment, 27 were lost to follow-up, consent was withdrawn for a further 8 and for 9, there were insufficient data for analysis (figure 1). Therefore,

Table 3 For children alive at 2 years (corrected age) outcomes at discharge according to availability of developmental outcome data and allocated group

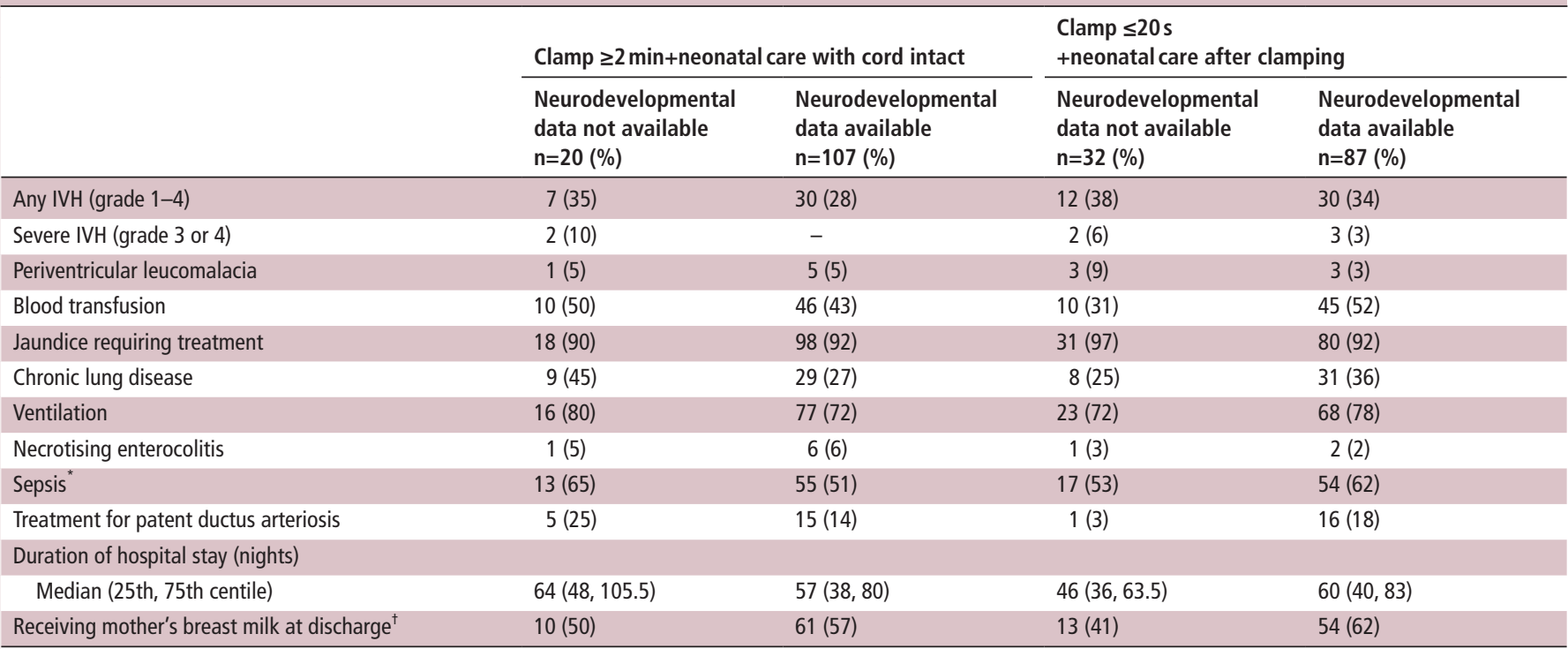

${ }^{*}$ Clinical sepsis defined as positive culture and $\geq 5$ days antibiotics or negative culture and $\geq 5$ days antibiotics.

tReceiving breast milk at discharge not known for two babies in the clamp cord $\geq 2$ min group with main outcome data available and one baby in both groups with no main outcome data available.

IVH, intraventricular haemorrhage. 


\begin{tabular}{|c|c|c|c|c|}
\hline & $\begin{array}{l}\text { Clamp } \geq 2 \text { min+neonatal care } \\
\text { with cord intact } n=115(\%)\end{array}$ & $\begin{array}{l}\text { Clamp } \leq 20 s+\text { neonatal care } \\
\text { after clamping } n=103(\%)\end{array}$ & $\begin{array}{l}\text { Risk difference } \\
(95 \% \mathrm{Cl})\end{array}$ & $\begin{array}{l}\text { Risk ratio } \\
(95 \% \mathrm{Cl})\end{array}$ \\
\hline Death or adverse neurodevelopmental outcome & $24(21)$ & $35(34)$ & $-13 \%(-25 \%$ to $-1 \%)$ & $0.61(0.39$ to 0.96$)$ \\
\hline Death & 8 (7) & $16(16)$ & $-9 \%(-17 \%$ to $0 \%)$ & $0.45(0.20$ to 1.00$)$ \\
\hline Adverse neurodevelopmental outcome & $16(14)$ & $19(18)$ & $-5 \%(-14 \%$ to $5 \%)$ & $0.75(0.41$ to 1.39$)$ \\
\hline \multicolumn{5}{|c|}{ For children alive at 2 years, type of adverse neurodevelopment outcome* } \\
\hline Motor & $2(2)$ & $5(6)$ & & \\
\hline Cognitive & $11(10)$ & $6(7)$ & & \\
\hline Hearing & $2(2)$ & $2(2)$ & & \\
\hline Speech/language & $9(8)$ & $14(16)$ & & \\
\hline Vision & $2(2)$ & $2(2)$ & & \\
\hline
\end{tabular}

*For: clamp $\geq 2$ min+neonatal care with cord intact $n=107$; clamp $\leq 20$ s+neonatal care after clamping $n=87$.

194 children were assessed at 2 years corrected age. As there were 24 deaths, 218 children were included in the analysis of the main outcome (115 allocated deferred clamping ( $\geq 2 \mathrm{~min}$ ); 103 allocated immediate clamping $(\leq 20 \mathrm{~s})$ ) (figure 1$)$.

\section{Baseline characteristics and outcomes to discharge}

Baseline characteristics and outcomes at discharge were similar for children included in the analysis of outcomes to discharge $(n=270)$ and those included in the analysis of outcomes at 2 years $(n=218)$ (table 1$)$. For children included in analysis of outcomes at 2 years, baseline characteristics remained balanced between the allocated groups, with a median gestation at birth of 29 weeks (table 1 ).

\section{Neurodevelopmental assessment}

Data were available for 194/238 (82\%) children alive at 2 years corrected age (table 2). Bayley-III data were available for 119/238 $(50 \%)$ children, with a higher percentage allocated deferred clamping $(66 / 124,53 \%)$ than immediate clamping $(53 / 114$, 46\%; table 2) being assessed. Corrected age at assessment was similar between groups. For 24 children with Bayley-III data, one or more scales were incomplete (13 deferred clamping, 11 immediate clamping); for 18 , this was the motor scale. Interrater reliability of Bayley-III assessments was excellent with 97\% agreement for the cognitive scale, 97\% for language and 100\% for motor (data not shown).

ASQ-3 data were available for 148/238 (62\%) children, again with a higher response for those allocated deferred clamping $(83 / 124,67 \%)$ than immediate clamping $(65 / 114,57 \%)$ (table 2$)$. For most children (138), the 24-month ASQ-3 was completed. The main reason for having neither Bayley-III nor ASQ-3 data was no response to the invitation to participate (72 children).

Routine clinical data were obtained for 39/238 (16\%) children with neither ASQ-3 nor Bayley-III data (20 deferred clamping, 19 immediate clamping; table 2).

For children allocated deferred clamping, there was some evidence that those lost to follow-up had poorer outcomes at discharge than those who were assessed (table 3). In this group, a greater percentage of children with no neurodevelopmental data had IVH, blood transfusion or chronic lung disease compared with those with data. This was not observed for children allocated immediate clamping where there was some evidence of the opposite trend (table 3).

\section{Death and adverse neurodevelopmental outcomes}

Of children allocated deferred clamping, 24/115 (21\%) died or had an adverse neurodevelopmental outcome compared with $35 / 103$ (34\%) allocated immediate clamping (table 4); RR 0.61 (95\% CI
0.39 to 0.96$)$; RD $-13 \%$ (95\% CI $-25 \%$ to $-1 \%)$. Using multiple imputation to account for loss to follow-up gave RR 0.69 (95\% CI 0.44 to 1.09$)$ and $\mathrm{RD}-9 \%(95 \% \mathrm{CI}-21 \%$ to $2 \%)$.

In the deferred clamping group, 8/115 (7\%) children died compared with $16 / 103(16 \%)$ in the immediate clamping group (table 4). Of these, 3 were stillborn (1 deferred clamping, 2 immediate clamping), 19 died before discharge from hospital (6 deferred clamping, 13 immediate clamping) and 2 died after discharge (one in each group). Of children alive at 2 years, 16/107 (15\%) allocated deferred clamping had an adverse neurodevelopmental outcome compared with 19/87 (22\%) allocated immediate clamping.

Of children with a neurodevelopmental assessment, the most common type of adverse outcomes were in speech/language and cognitive impairment (table 4). Summary statistics for the 24-month ASQ-3 and Bayley-III are available in online supplementary appendix 2 .

\section{DISCUSSION}

Follow-up of children in the Cord Pilot Trial at 2 years corrected age suggests that deferring cord clamping for at least 2 min and providing immediate neonatal care, if needed, with cord intact may reduce the risk of death or adverse neurodevelopmental outcome compared with immediate clamping $(\leq 20 \mathrm{~s})$ and neonatal care after clamping. However, these results were sensitive to imputation to account for missing data. More children allocated deferred clamping had neurodevelopmental assessment data available; however, these children appeared to have fared slightly better at hospital discharge than those with missing follow-up data. In the immediate clamping group, there was evidence of the opposite trend when outcomes at discharge were compared according to availability of neurodevelopmental assessment data. Since the trial was not powered to demonstrate clinically important differences in outcome, confirmation is required in large high-quality randomised trials.

Strengths of our trial were that clamping was deferred for longer than in other trials at very preterm birth and that immediate neonatal care (including stabilisation and resuscitation), if needed, was provided with the cord intact. ${ }^{20}$ Providing neonatal care with the cord intact allowed high-risk babies needing immediate resuscitation at birth to be randomised, a group largely excluded from previous trials. ${ }^{21}$

A composite of death and adverse neurodevelopment was chosen as the main outcome, a decision supported by our parent representatives as being relevant to parents. Despite the problems of composite outcomes, this is considered acceptable for perinatal and neonatal trials provided it is likely that the direction of effect is the same for all components of the composite ${ }^{22}$ 
as is the case for alternative policies for cord clamping. In our trial, the overall reduction in death or adverse neurodevelopment in the deferred clamping group was primarily due to the reduction in death before discharge. There was no clear evidence of a difference between groups in adverse neurodevelopmental outcome or death after discharge.

Achieving high response rates for long-term follow-up is necessary to ensure sample representation; however, this is challenging. In order to achieve our $82 \%$ response rate, this necessitated employing multiple approaches and combining results obtained from different assessment tools, including diagnostic tests (Bayley-III), parent questionnaires (ASQ-3) and data obtained from routine clinical assessments. As screening tests may have poor diagnostic accuracy compared with gold standard tests ${ }^{23}$ and routine clinical assessments have poor sensitivity for evaluating cognitive outcomes in this population, ${ }^{24}$ this approach represents a limitation of our study. Future trials should attempt to attain long-term follow-up rates in excess of $90 \%$ and use a single standardised test to assess neurodevelopmental outcomes. Lower follow-up for children allocated immediate clamping may have been due to the women feeling they were not part of the trial as they received usual care or being disappointed they did not receive the 'intervention' of deferred clamping. ${ }^{25}$

Using routine data for developmental assessment in perinatal trials merits further evaluation as it is less intrusive for parents and less costly in terms of resources. In the UK, the National Institute for Health and Care Excellence has recently introduced guidance that high-risk children, such as those born before 30 weeks' gestation, are eligible for enhanced developmental surveillance. This includes a developmental assessment at 2 years corrected age ${ }^{26}$ Provided they are of sufficient quality, these data could be used to determine neurodevelopmental outcomes for children in future trials.

The most recent systematic review, which includes the Cord Pilot Trial, concluded that delayed clamping reduces hospital mortality compared with immediate clamping, with no clear effect on serious neonatal morbidity. ${ }^{7}$ This review emphasises the importance of follow-up for children recruited to both existing and future trials. The latter should be large enough to provide adequate power to detect clinically important differences in outcomes and will also need to achieve high follow-up rates to enable reliable comparisons of neurodevelopmental outcomes. Since providing robust evidence is likely to require multiple trials, a systematic review and individual participant data meta-analysis of cord management at preterm birth is underway. ${ }^{27}$

\section{CONCLUSIONS}

Follow-up of children in the Cord Pilot Trial suggests deferred clamping with immediate neonatal care, if needed, beside the mother may reduce the risk of death or adverse neurodevelopmental outcome at 2 years corrected age compared with immediate clamping and neonatal care after clamping. Large high-quality trials are needed to confirm the true benefits and harms.

Acknowledgements The authors would like to thank all children and women who participated in this trial along with their families and the clinical and research staff at the sites. The authors would also like to thank Bernard Schoonakker for participating in the Blinded Endpoint Review Committee along with Gill Gyte (National Childbirth Trust) and Zoe Chivers (Bliss) for their advice and input.

Contributors The trial was conceived by LD and Gill Gyte. The protocol, trial procedures and strategies for recruitment and consent were developed in consultation with a broad range of stakeholders including parent representatives, clinicians, researchers, National Institute for Health Research (NIHR) preterm birth programme grant steering group, independent Trial Steering Committee, Data Monitoring Committee and the Nottingham Clinical Trials Unit. The paper was drafted by LA-B with input and comments from other authors. All authors have read and agreed the final paper.
Funding This trial is independent research funded by the NIHR under its Programme Grants for Applied Research funding scheme (RPPG0609-10107).

Disclaimer The views expressed are those of the authors and not necessarily those of the NHS, the NIHR or the Department of Health. The funder had no role in study design, conduct, analysis or reporting.

Competing interests JD reports grants from NIHR during the conduct of the trial; LD reports memberships to CTUs funded by NIHR. All other authors have nothing to disclose.

Patient consent for publication Not required.

Ethics approval Nottingham REC 2 (NRES reference 12/EM/0283).

Provenance and peer review Not commissioned; externally peer reviewed.

Open access This is an open access article distributed in accordance with the Creative Commons Attribution 4.0 Unported (CC BY 4.0) license, which permits others to copy, redistribute, remix, transform and build upon this work for any purpose, provided the original work is properly cited, a link to the licence is given, and indication of whether changes were made. See: https://creativecommons.org/ licenses/by/4.0/.

\section{ORCID iDs}

Lindsay Armstrong-Buisseret http://orcid.org/0000-0002-8045-5464

Lelia Duley http://orcid.org/0000-0001-6721-5178

\section{REFERENCES}

1 Manktelow BN, Smith LK, Prunet C, et al. MBRACE-UK Perinatal Mortality Surveillance Report, UK Perinatal Deaths for Births from January to December 2015. Leicester: The Infant Mortality and Morbidty Studies, Department of Health Sciences, University of Leicester, 2017

2 Johnson S, Marlow N. Early and long-term outcome of infants born extremely preterm. Arch Dis Child 2017;102:97-102.

3 Saigal S, Doyle LW. An overview of mortality and sequelae of preterm birth from infancy to adulthood. Lancet 2008;371:261-9.

4 Linsell L, Johnson S, Wolke $D$, et al. Cognitive trajectories from infancy to early adulthood following birth before 26 weeks of gestation: a prospective, populationbased cohort study. Arch Dis Child 2018;103:363-70.

5 Eryigit Madzwamuse S, Baumann N, Jaekel J, et al. Neuro-cognitive performance of very preterm or very low birth weight adults at 26 years. J Child Psychol Psychiatry 2015:56:857-64.

6 Rabe H, Diaz-Rossello JL, Duley L, et al. Effect of timing of umbilical cord clamping and other strategies to influence placental transfusion at preterm birth on maternal and infant outcomes. Cochrane Database Syst Rev 2012:8:Cd003248.

7 Fogarty M, Osborn DA, Askie L, et al. Delayed vs early umbilical cord clamping for preterm infants: a systematic review and meta-analysis. Am J Obstet Gynecol 2018;218:1-18.

8 Duley L, Dorling J, Pushpa-Rajah A, et al. Randomised trial of cord clamping and initial stabilisation at very preterm birth. Arch Dis Child Fetal Neonatal Ed 2018;103:F6-14.

9 Pushpa-Rajah A, Bradshaw L, Dorling J, et al. Cord pilot trial - immediate versus deferred cord clamping for very preterm birth (before 32 weeks gestation): study protocol for a randomized controlled trial. Trials 2014;15:258.

10 Bradshaw LE, Pushpa-Rajah A, Dorling J, et al. Cord pilot trial: update to randomised trial protocol. Trials 2015;16:407.

11 Squires J, Twombly E, Bricker D, et al. Ages \& Stages Questionnaires, Third Edition (ASQ-3) User's Guide. Baltimore, MD: Brookes Publishing, 2009

12 Bayley N. Bayley Scales of Infant and Toddler Development. 3rd edn. San Antonio, Texas: Pearson, 2005.

13 Johnson S, Evans TA, Draper ES, et al. Neurodevelopmental outcomes following late and moderate prematurity: a population-based cohort study. Arch Dis Child Fetal Neonatal Ed 2015:100:F301-8.

14 Anderson PJ, De Luca CR, Hutchinson E, et al. Underestimation of developmental delay by the new Bayley-III Scale. Arch Pediatr Adolesc Med 2010;164:352-6.

15 Johnson S, Moore T, Marlow N. Using the Bayley-III to assess neurodevelopmental delay: which cut-off should be used? Pediatr Res 2014;75:670-4.

16 British Association of Perinatal Medicine. Report of a BAPM/RCPCH Working Group. Classification of Health Status at 2 years as a perinatal outcome. London: BAPM, 2008.

17 White IR, Royston P, Wood AM. Multiple imputation using chained equations: Issues and guidance for practice. Stat Med 2011;30:377-99.

18 Gill B. The English Indices of Deprivation London: Department for Communities and Local Government. 2015. Available: https://www.gov.uk/government/statistics/ english-indices-of-deprivation-2015 [Accessed 19 Nov 2018].

19 Carpenter JR, Kenward MG. Missing data in randomised controlled trials: a practical guide. London: London School of Hygiene \& Tropical Medicine, 2007.

20 Batey N, Yoxall CW, Fawke JA, et al. Fifteen-minute consultation: stabilisation of the high-risk newborn infant beside the mother. Arch Dis Child Educ Pract Ed 2017;102:235-8. 


\section{Original research}

21 Wyllie J, Bruinenberg J, Roehr CC, et al. European Resuscitation Council Guidelines for Resuscitation 2015: Section 7. Resuscitation and support of transition of babies at birth. Resuscitation 2015;95:249-63.

22 Marlow N. Is survival and neurodevelopmental impairment at 2 years of age the gold standard outcome for neonatal studies? Arch Dis Child Fetal Neonatal Ed 2015;100:F82-F84.

23 Johnson S, Marlow N. Developmental screen or developmental testing? Early Hum Dev 2006;82:173-83.

24 Wong HS, Cowan FM, Modi N. Medicines for Neonates Investigator Group. Validity of neurodevelopmental outcomes of children born very preterm assessed during routine clinical follow-up in England. Arch Dis Child Fetal Neonatal Ed 2018;103:F479-84.
25 Sawyer A, Chhoa C, Ayers S, et al. Women's views and experiences of two alternative consent pathways for participation in a preterm intrapartum trial: a qualitative study. Trials 2017;18:422.

26 National Institute for Health and Care Excellence. Developmental follow-up of children and young people born preterm: NICE Quality Standard London, 2018. Available: https://www.nice.org.uk/guidance/qs169 [Accessed 13 Nov 2018].

27 Duley L, Askie L, Yang M. Cord clamping and placental transfusion at preterm birth prospective meta-analysis. PROSPERO 2013:CRD42013004405. Available: http:// www.crd.york.ac.uk/PROSPERO/display_record.php?ID=CRD42013004405 [Accessed 13 Nov 2018]. 